\title{
The Structure and the Growth of Guinier-Preston Zones in an Al-6.8 at\% Zn Alloy*
}

\author{
By Masanori Murakami**, Masahiko Morinaga**, \\ Osamu Kawano*** and Yotaro Murakami***
}

\begin{abstract}
A study by means of an X-ray small angle scattering method was carried out on the structure and the growth of GuinierPreston zones in a binary aluminum-6.8 at\% zinc alloy. By the isothermal measurement of scattering intensities of the alloy aged at $40^{\circ} \mathrm{C}$, it was found that both models for the structure of G. P. zones proposed by Walker and Guinier ${ }^{(1)}$ and by Gerold and Schweizer ${ }^{(2)}$ were not suitable at the early stages of aging, but that the latter model was suitable at the later stages. Moreover, the mechanism of growth of these zones was indicated to be accordant with the diffusion-controlled Ostwald ripening. (Received February 28, 1970)
\end{abstract}

\section{Introduction}

The studies by X-ray diffraction and electron microscopy gave the evidence that G. P. zones of a binary aluminum-rich zinc alloy were stable when aged at low temperatures after a quench from homogenization temperatures $^{(3) \sim(5)}$.

For the structure of G. P. zones two models. (Models 1 and 2) have been proposed. They are schematically represented in Fig. 1. Model 1 is the structure of zones

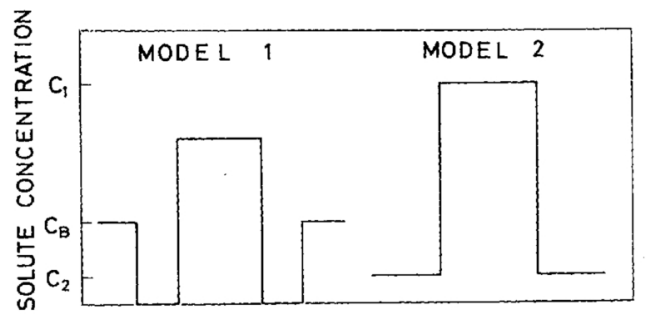

DISTANCE

Fig. 1 Two models for the structure of G.P. zones. Model 1 consists of solute-rich zones surrounded by a solutedepleted shell with the rest of the matrix unchanged ${ }^{(1)}$ and Model 2 is a conventional dispersion of zones in a matrix (2). $C_{1}$ and $C_{2}$ are the solute concentrations inside and outside G.P. zones, respectively and $C_{B}$ is the alloy concentration.

of solute atoms surrounded by a shell denuded in solute with the matrix remaining supersaturated at the original composition of the alloy, which was chosen by Walker and Guinier ${ }^{(1)}$ to explain the appearance of the maximum in X-ray small angle scattering curves in an Al-Ag alloy. This model was also applied to the zones in an Al-Zn system. However, Gerold and Schweizer ${ }^{(2)}$ pointed out

* This paper was presented at the Autumn Meeting of the Japan Institute of Metals, October, 1969, Hiroshima, Japan.

** Graduate School, Kyoto University, Kyoto, Japan.

*** Department of Metallurgy, Faculty of Engineering, Kyoto University, Kyoto, Japan.

(1) C. B. Walker and A. Guinier : Acta Met., 1(1953), 568.

(2) V. Gerold and W. Schweizer : Z. Metallk., 52 (1961), 76.

(3) W. Merz und V.Gerold : Z. Metallk., 57 (1966), 607.

(4) M. Simerská and V. Syneček : Acta Met.,15 (1967), 223.

(5) T. Niklewski, P.Spiegelberg and K. Sunbulli : Metal Sci. J., 3 (1969), 23.

Trans. J I M that this model was inconsistent with the fact that the integrated intensity calculated from the scattering intensity was constant during the course of isothermal aging. Hence, a new model was given (Model 2): zones of solute concentration $C_{1}$ were distributed at random in the matrix of $C_{2}$, where $C_{1}$ and $C_{2}$ are the solute concentrations determined from the metastable miscibility gap at a given temperature. This model was also known to be suitable even for the early stages of aging. Recently, by measuring the scattering intensity of Al-Zn alloys isothermally aged at $-45^{\circ} \mathrm{C}$, Bonfiglioli and Guinier ${ }^{(6)}$ indicated that though Model 2 was suitable for the later stages of aging, it was contradictory at the initial stages of decomposition.

In the present paper, to examine the structure of G.P. zones throughout aging the scattering intensity of an aluminum alloy containing 6.8 at \% zinc aged isothermally at $40^{\circ} \mathrm{C}$ was measured by means of an X-ray small angle scattering method. Although the measured intensity provides a variety of information, by examining the integrated intensity ${ }^{(7)}$, applying Porod's law ${ }^{(8)}$ and calculating the short-range order parameter ${ }^{(9)}$, which were analyzed from the scattering curves, we attempted to indicate the change of sollute concetrations inside and outside G.P. zones, to show the gradient of solute concentrations at the interface between zones and matrix and to clarify the distribution of solute atoms in the alloy. (In the present case, the interference function ${ }^{(10)}$ among zones is neglected.)

The initial decomposition of the supersaturated solid solution proceeds either by the spinodal or nucleation and growth mechanism. In a binary Al-22 at $\% \mathrm{Zn}$ alloy Rundman and Hilliard ${ }^{(11)}$ demonstrated that the change of the scattering intensity at $65^{\circ} \mathrm{C}$ aging coincided with the spinodal theory developed by Hillert ${ }^{(12)}$

(6) A. F. Bonfiglioli et A. Guinier : Acta Met., 14(1966), 1213

(7) A. Guinier and G. Fournet : Small Angle Scattering of $X$ rays, John Wiley, New York, (1955), p. 18.

(8) G.Porod : Kolloidzschr.,124 (1951), 83.

(9) A. Guinier : X-Ray Diffraction, Freeman, (1963), p. 264.

(10) A. Guinier and G. Fournet : Small Angle Scattering of $X-$ rays, John Wiley, New York, (1955), p. 33.

(11) R. R. Rundman and J. E. Hilliard : Acta Met., 15(1967), 1025.

(12) M. Hillert : Acta Met., 9(1961), 525. 
and $\mathrm{Cahn}^{(13)}$, and the present authors ${ }^{(14)}$, though the calculated spinodal temperature is below $0^{\circ} \mathrm{C}^{(11)}$, estimated the spinodal temperature to be approximately $120^{\circ} \sim 130^{\circ} \mathrm{C}$ in an alloy containing 6.8 at $\%$ zinc. However, $\mathrm{Cook}^{(15)}$ has recently reported that the gradual shift in the intensity maximum during the isothermal aging makes it impossible to describe clustering kinetics. Besides, de Fontaine ${ }^{(16)}$ pointed out that there is no discontinuity either in morphology or in kinetics at the spinodal. Thus, the discussion on the kinetics of the formation of G.P. zones is not given here.

Lifshitz and Slyozov ${ }^{(17)}$ and Wagner ${ }^{(18)}$ established a theory for the growth of particles based on the diffusion equations. The validity of this theory was confirmed experimentally by Ardell ${ }^{(19)}$ in a binary $\mathrm{Ni}-\mathrm{Al}$ alloy. At stages where the solid solution is completely decomposed to the expected points of metastable miscibility gap, the diffusion coefficient of solute atoms can be evaluated from the slope of a plot of $\bar{R}^{3}(\bar{R}$ : average zone radius) and $t$ (time) by applying this theory to the zone growth. The validity of applicability of this theory to the zone growth will be given in the section of (III-2).

\section{Theoretical considerations}

\section{(1) Integrated intensity}

For the study of the as-quenched state and the time dependence of the solute concentrations inside and outside G. P. zones the investigation of the integrated intensity is utilized.

From the theory of small angle scattering ${ }^{(7)}$ the integrated intensity $Q_{0}$ was given as the function of the local fluctuation of electron density $\left(\rho-\rho_{0}\right)^{2}$,

$$
Q_{0}=2 \pi \int_{0}^{\infty} s J(s) \mathrm{d} s=\frac{\overline{\left(\rho-\rho_{0}\right)^{2}}}{\rho_{0}}
$$

where $J(s)$ is the observed scattering intensity when the line collimation is used for the primary beam, $s=2 \sin \theta / \lambda$. $\simeq 2 \theta / \lambda(2 \theta$ is the scattering angle and $\lambda$ is the wavelength of $\mathrm{X}$-ray used) and $\rho_{0}$ is the average electron density. Before the decomposition is completed, the value of $\left(\overline{\left.\rho-\rho_{0}\right)^{2}}\right.$, i. e. $Q_{0}$, should increase with time $t$. When the decomposition has been progressed to the points of metastable miscibility gap ${ }^{(20)}, Q_{0}$ should remain constant. In this case eq.(la) can be rewritten in terms of the solute concentrations inside $\left(C_{1}\right)$ and outside $\left(C_{2}\right)$ G. P. zones $^{(21)}$,

$$
Q_{0}=p\left(C_{1}-C_{B}\right)\left(C_{B}-C_{2}\right) \frac{\left(Z_{A}-Z_{B}\right)^{2}}{V_{a}}
$$

(13) J. W. Cahn : Acta Met., 9(1961), 795.

(14) M. Murakami, O. Kawano, Y. Murakami and M. Morinaga : Acta Met.,17(1969), 1517.

(15) H. E Cook : J. Phys. Chem. Solids, 30 (1969), 2427.

(16) D. de Fontaine : Private communication.

(17) I. M. Lifshitz and V. V. Slyozov : J. Phys. Chem. Solids, 19 (1961), 35

(18) C. Wagner : Z. Elektrochem., 65 (1961), 581.

(19) A.J. Ardell : The Mechanism of Phase Transformations in Crystalline Solids, Inst. Metals, London, (1969), p. 111.

(20) V. Gerold : Small-Angle X-ray Scattering, Gordon and Breach, New York, (1968), p. 299.

(21) V. Gerold : Phys. Stat. Sol.,1(1961), 37. where $C_{B}$ is the solute concentration of the alloy, $Z_{A}$ and $Z_{B}$ are the respective atomic numbers of the solute and solvent atoms, $V_{a}$ is the average atomic volume and $p$ is the fraction of the alloy which has segregated into zones.

\section{(2) Porod's law}

To investigate the gradient of the concentration of solutes at the interface of G. P. zones with average electron density $\bar{\rho}$, Porod's equation ${ }^{(8)}$ can be applied :

$$
\lim _{s \rightarrow \infty} s^{3} J(s)=\frac{S}{16 \pi^{3}}\left(\bar{\rho}-\rho_{0}\right)^{2}
$$

where $S$ is the total surface of G. P. zones per unit mass. According to this equation if there is a sharp interface between the two phases the value of $s^{3} J(s)$ should become nearly constant for large $s$.

\section{(3) Short-range order parameter}

The investigation of the short-range order parameter $\alpha$ gives the information on the distribution of solute atoms in the alloy. This parameter is defined by the probability $n_{B B}(x)$ that a pair of atoms separated by a distance $x$ is formed by two $\mathrm{Zn}$ atoms as follows ${ }^{(9)}$ :

$$
\alpha(x)=1-\frac{n_{B A}}{C_{A}}=1-\frac{1-n_{B B}}{C_{A}}=\frac{n_{B B}-C_{B}}{C_{A}}
$$

where

$$
\begin{gathered}
n_{B A}=\frac{N_{B A}}{N_{B B}+N_{B A}}, \quad n_{B B}=\frac{N_{B B}}{N_{B B}+N_{B A}}, \\
C_{A}=N_{A B}+N_{A A}, \quad C_{B}=N_{B B}+N_{B A} .
\end{gathered}
$$

$N_{A A}, N_{A B}, N_{B B}$ and $N_{B A}$ are the proportions of the various types of pairs on the vector $x$. Since the knowledge of the order parameter determines completely the scattering pattern, the order parameter can inversely be determined from the scattering pattern as follows:

$$
\alpha(x)=\frac{1}{2 \pi x} \frac{\int_{0}^{\infty} I(s) \sin (2 \pi x s) s \mathrm{~d} s}{\int_{0}^{\infty} I(s) s^{2} \mathrm{~d} s}
$$

where $\alpha(x)$ is normalized as $\alpha(0)=1$, and $I(s)$ is the scattering intensity after beam height correction.

\section{(4) Growth law}

When the solute concentration in the solid solution has almost reached its equilibrium value, the LifshitzWagner theory ${ }^{(17)(18)}$ is applicable to spherical G.P. zones dispersed in the matrix.

The theoretical kinetic equation for G. P. zones growth is,

$$
\bar{R}^{3}-\bar{R}_{0}^{3}=k\left(t-t_{0}\right)
$$

where $\vec{R}$ and $\widetilde{R}_{0}$ are the average zone radius at time $t$ and at the onset. of coarsening $\left(t_{0}\right)$ respectively. The rate constant $k$ is given by

$$
k=\frac{2 \sigma D C_{e} V_{m}^{2}}{\rho_{c}^{2} R T},
$$

where $D$ is the diffusion coefficient of the solute, $V_{m}$ is the molar volume of the zone, $C_{e}$ is the concentration 
of solute in equilibrium with a particle of infinite size, $\rho_{c}$ is a numerical constant related to the distribution of zone sizes (theoretically $\rho_{c}=3 / 2$ ), $R T$ has its usual meaning, and $\sigma$ is the interfacial free energy of the zonematrix interface.

$\sigma$ is calculated on the basis of the situations in which G. P. zones and matrix are random solid solutions and the boundary is coherent. The interfacial energy is written by the summation of the structural interfacial energy $\Delta F^{\text {str }}$ and the chemical interfacial energy $\Delta F^{\text {chem }}$. In the case of the coherent zone-matrix boundary $\Delta F^{\text {str }} \simeq 0$.

Then,

$$
\sigma \simeq \Delta F^{\text {chem }}=\Delta H^{\text {chem }}-T \Delta S^{\text {chem }}
$$

To calculate the enthalpic component $\Delta H^{\text {chem }}$ of the chemical interfacial energy, the relationship of Servi and Turnbull ${ }^{(22)}$ can be applied to the zone-matrix boundary when the small misfit across the boundary is ignored :

$$
\Delta H^{\text {chem }}=N_{s} Z_{s}\left(C_{1}-C_{2}\right)^{2} \Delta H_{m}
$$

where $N_{s}$ is the number of atoms per unit area in the plane of the interface, $Z_{s}$ is the surface coordination number, and $\Delta H_{m}$ is the mixing enthalpy.

The entropic component $\Delta S^{\text {chem }}$ of the chemical interfacial energy is defined by,

$$
\Delta S^{\mathrm{chem}}=S_{m z}-\frac{1}{2}\left(S_{m m}+S_{z z}\right)
$$

where $S_{m m}, S_{z z}$ and $S_{m z}$ are the positional entropies at the fully coherent matrix-matrix, zone-zone and matrix-zone boundaries, respectively, $\Delta S^{\text {chem }}=0$ because the juxtaposition of two random solid solution produces an interphase boundary at which the randomness of the bond arrangements is exactly the average randomness at crystallographically identical boundaries within the component phases. Hence,

$$
\sigma \simeq \Delta H^{\text {chem }} .
$$

\section{Experimental Procedures}

The material used was an aluminum- 6.8 at $\%$ zinc alloy prepared from $99.99 \%$ aluminum and $99.99 \%$ zinc. The measurements of $\mathrm{X}$-ray small angle scattering were carried out with the foils of $0.2 \mathrm{~mm}$ thick using Ni-filtered $\mathrm{CuK}_{\alpha}$ radiation. The specimens were homogenized at $300^{\circ} \mathrm{C}$ for one hour and cooled rapidly in a $0^{\circ} \mathrm{C}$ water bath placed below the furnace. After holding at this temperature for $15 \mathrm{sec}$, they were further cooled to the liquid nitrogen temperature before aging. The aging was performed in an air furnace kept at $40^{\circ} \mathrm{C}$ $\pm 0.1^{\circ} \mathrm{C}$. The heat-up time of the specimens was less than 10 sec. The scattering intensity was counted at liquid nitrogen temperature at $1 / 8^{\circ}$ intervals in the scattering angle $(2 \theta)$ up to $2 \theta_{0}=3.75^{\circ}$ with line collimation. At the angles higher than $2 \theta_{0}$, the $(2 \theta)^{-3}$ law was utilized. The counted intensity spectra were corrected for beam height using a technique of the computer

(22) I. Servi and D. Turnbul] : Acta Met., 14 (1966), 161.

(23) P. W. Schmidt : Acta Cryst., 19(1965), 938 method developed by Schmidt ${ }^{(23)}$. The calculation of $Q_{0}$ was carried out by the method developed by Gerold ${ }^{(21)}$ and the average zone radius was obtained by using Guinier's approximation $^{(24)}$ utilizing the slope of a $\log I$ vs. $(2 \theta)^{2}$ plot of X-ray scattering intensity data. In evaluating $\alpha(x)$ of eq. (4), the computer method was also used setting an upper limit $2 \theta=7.5^{\circ}$.

\section{Experimental Results and Discussions}

\section{Structure of G. P. zones}

Representative X-ray small angle scattering curves for specimens which were quenched from $300^{\circ} \mathrm{C}$ and aged at $40^{\circ} \mathrm{C}$ for the specified times are shown in Fig. 2. These curves were analyzed by utilizing the integrated intensity, Porod's law and the short-range order parameter.

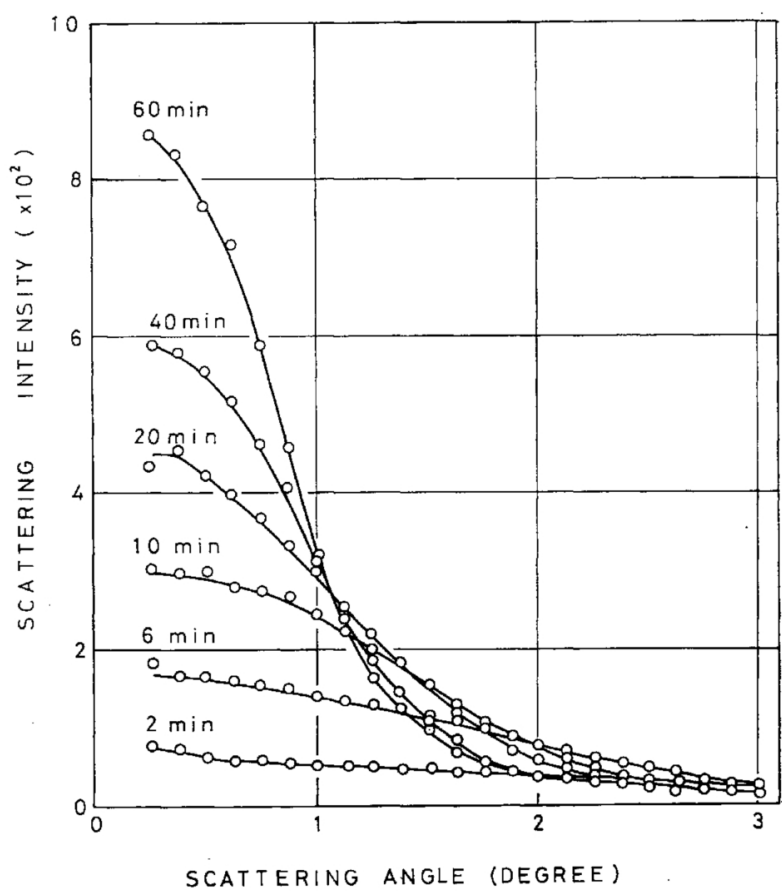

Fig. $2 \mathrm{X}$-ray intensities scattered from the alloys quenched from $300^{\circ} \mathrm{C}$ and aged for the specific times at $40^{\circ} \mathrm{C}$.

\section{(1) Integrated intensity}

Using eq. (1a) the integrated intensity $Q_{0}$ was calculated as shown in Fig. 3. The values of $Q_{0}$ in the specimens of the as-quenched state and aged for 1 min cannot be obtained, because the measured intensities were too weak to find the critical value of $s$, where $\mathrm{d} Q_{0} / \mathrm{d} s \simeq 0$. After $2 \min Q_{0}$ increases as aging proceeds up to the critical time $t_{0}(\sim 6 \mathrm{~min})$ and after $t_{0}$ it becomes constant within experimental accuracy. From eq. (la) this increase suggests the increase in the degree of discrepancy of electron densities inside and outside G.P. zones. At $t_{0}$ it is made clear by eq. (1b) that the decomposition has been progressed to the points of the metastable miscibility gap ${ }^{(20)}$, that is, the solute concentrations inside and outside G. P. zones have been progressed to $C_{1}$ and

(24) A. Guinier and G. Fournet : Small Angle Scattering of $X$ rays, John Wiley, New York, (1955), p. 24. 


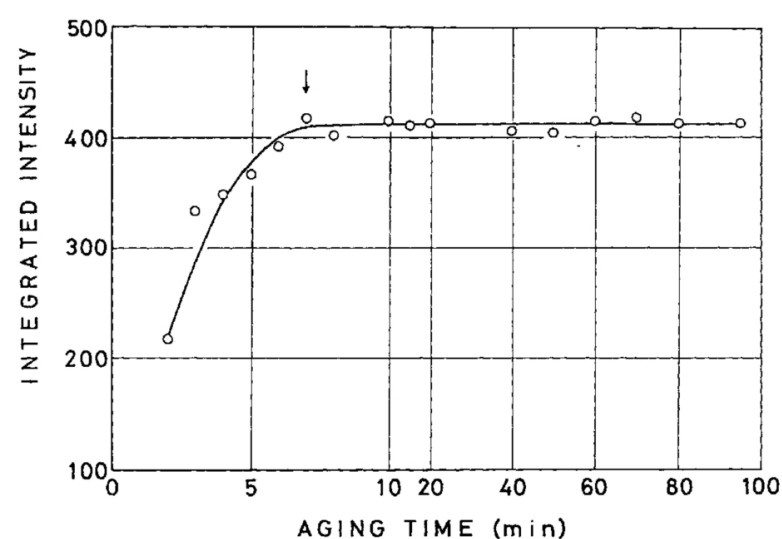

Fig. 3 Change of the integrated intensities of the alloys quenched from $300^{\circ} \mathrm{C}$ and aged at $40^{\circ} \mathrm{C}$.

$C_{2}$ respectively, where $p$ is considered to be unity. The constancy of $Q_{0}$ after $t_{0}$ means that the values of $C_{1}$ and $C_{2}$ do not change during subsequent aging, although the zone radius increases with aging time.

\section{(2) Porod's law}

As given in eq. (2), the plot of $s^{3} J(s)$ vs. $s^{3}$ gives the qualitative information on the gradient of solute concentration at the interface of G. P. zones. This plot is shown in Fig. 4 for the specimens aged for various times. In the early stages of aging $s^{3} J(s)$ increases monotonously, but after $6 \mathrm{~min}$ it becomes constant for large $s$, obeying Porod's law. These results mean that $G$. P. zones having a sharp interface with the matrix are formed after $6 \mathrm{~min}$ and that the interface is not sharp until this critical time. It is interesting to note that this critical time almost coincides with the times when the integrated intensity shows a constant value.

\section{(3) Short-range order parameter}

Using eq. (4) the short-range order parameter $\alpha(x)$ can be calculated from the scattering intensities in Fig. 2. From these calculated $\alpha(x)$, the probability $n_{B B}(x)$ that a

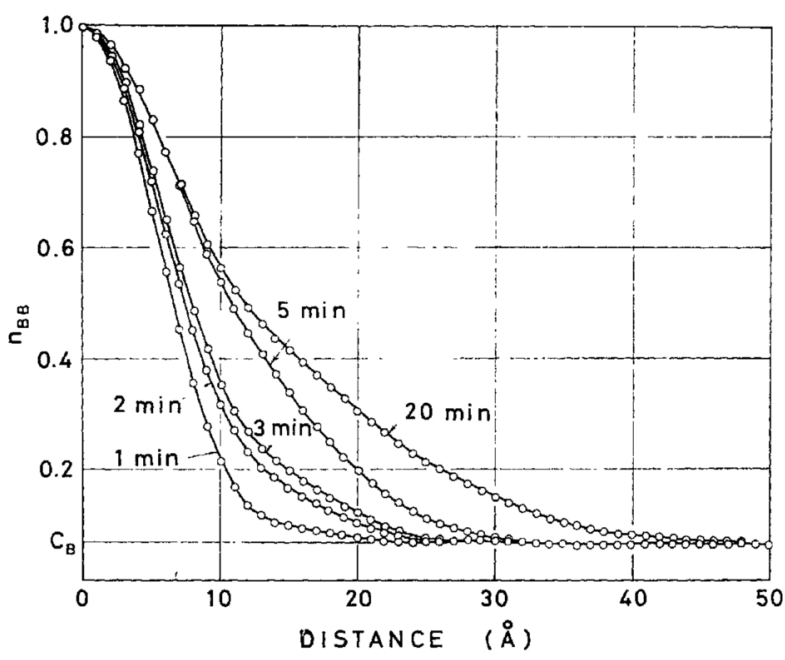

Fig. 5 Probability of finding a zinc atom at a distance $x$ from a given zinc atom for the alloys quenched from $300^{\circ} \mathrm{C}$ and aged at $40^{\circ} \mathrm{C}$.

pair of atoms separated by a distance $x$ is formed by two $\mathrm{Zn}$ atoms can be obtained as shown in Fig. 5 .

The fact that $n_{B B}$ is higher than the average $C_{B}$ over distances of $20 \sim 30 \AA$ demonstrates the existence of segregated regions of atoms at this aging temperature. As aging proceeds these regions become larger and it is noted that the regions where $n_{B B}$ is lower than the average value cannot be found even at the initial aging as in the case of the $\mathrm{Al}-\mathrm{Ag}^{(4)}$ or $\mathrm{Cu}-\mathrm{Ni}^{(25)}$ system.

\section{(4) New model}

It is found that these results cannot be interpreted by supposing the structure of G. P. zones as only Model 1 or 2 throughout aging.

Model 1 is applicable mainly for the initial stages of aging on condition that at the aging times less than $t_{0}$ the following conditions are fulfilled : (1) $Q_{0}$ is lower than the final value $Q_{f},(2)$ Porod's law is not satisfied

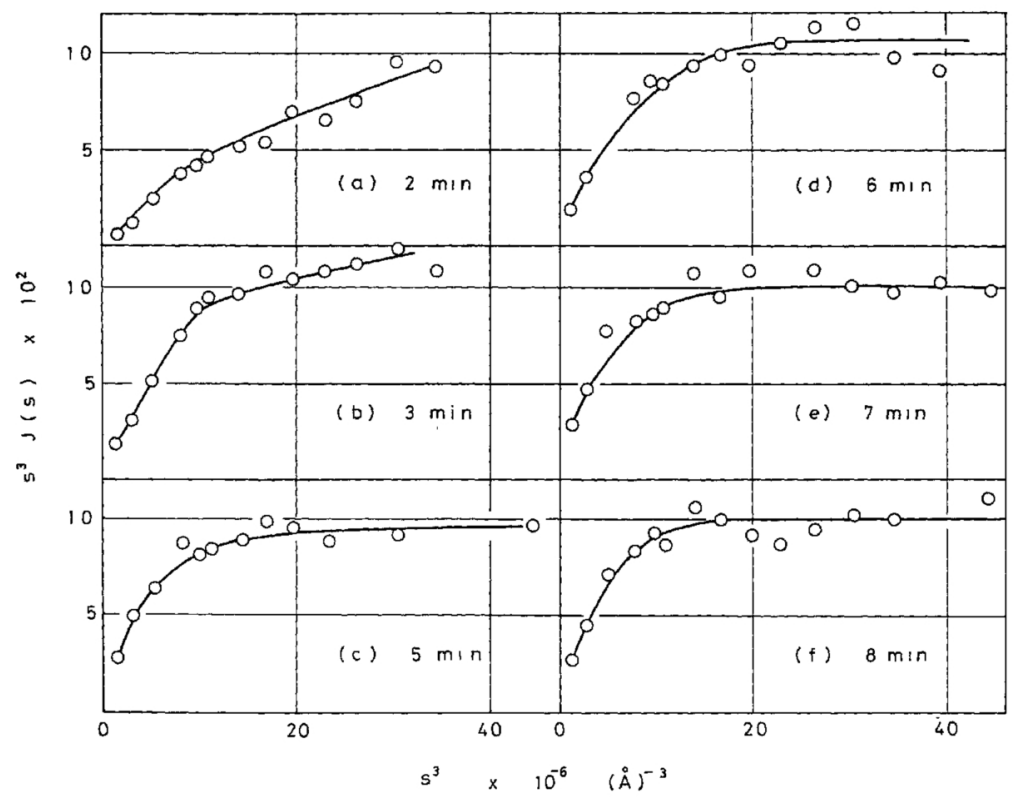

Fig. 4. Variation of $s^{3} J(s)$ as a function of $s^{3}$ for the alloys quenched from $300^{\circ} \mathrm{C}$ and aged at $40^{\circ} \mathrm{C}$.

(25) A. Kidron : Phys. Rev. Letters, 22 (1969), 774. 
and (3) the region where $n_{B B}$ is lower than $C_{B}$ is observed. Though conditions (1) and (2) were satisfied at times less than $t_{0}$, condition (3) was not. Besides, after $t_{0}$ all of these conditions were no longer valid. This suggests that Model I may not be valid even at the initial stages of aging.

In the case of Model 2, the following conditions are required throughout aging : (1) $Q_{0}$ is constant, (2) Porod's law is satisfied and (3) $n_{B B}$ is higher than $C_{B}$. As shown in Figs. 3 and 4 , at the initial stages $\left(t<t_{0}\right)$ though condition (3) was valid, conditions (1) and (2) were not satisfied. However, at the later stages $\left(t>t_{0}\right)$ these conditions were shown to be satisfactory. Thus, Model 2 is considered improper at the initial stages, but it may be suitable for the structure of $G$. P. zones at the later stage.

Therefore, at the later stages Model 2 is considered to be consistent with the present results, but at the initial stages both models are inconsistent. Thus a new model (Model 3) at the initial stage is given in Fig. 6. At this

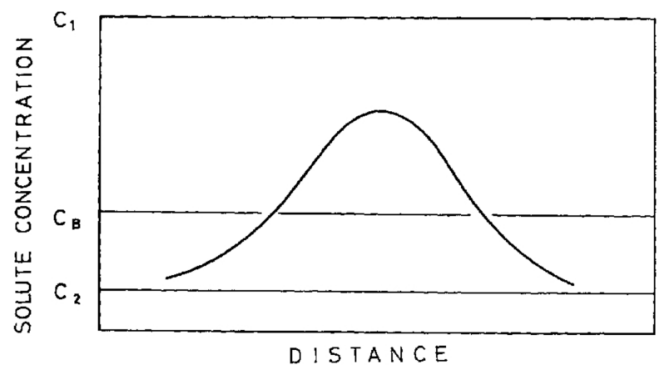

Fig. 6 Proposed model for the structure of G.P. zones at the early stages of aging.

stage the solute concentration $\left(C_{1}{ }^{\prime}\right)$ inside G. P. zones does not reach the equilibrium value $C_{1}$, the concentration of the matrix is still supersaturated $\left(C_{2}<C_{2}{ }^{\prime}\right)$ and the gradient of solute concentration at the interfaces between zones and matrix is not sharp. Recently de Fontaine ${ }^{(16)}$ indicated that this model is the same as the one ${ }^{(26)}$ simulated by computer inside spinodal. As aging proceeds, both $C_{1}{ }^{\prime}$ and $C_{2}{ }^{\prime}$ tend to approach $C_{1}$ and $C_{2}$ respectively and at $t_{0} C_{1}{ }^{\prime}=C_{1}$ and $C_{2}{ }^{\prime}=C_{2}$. The gradient of concentration becomes sharp simultaneously. At $t_{0}$ the decomposition is completed and after $t_{0}$ these zones grow keeping the solute concentrations both inside and outside the zones constant. This model is not contradictory to the present results.

\section{Kinetics of growth of G.P. zones}

The law of diffusion-controlled Ostwald ripening is applicable only to the growth of particles whose structures and solute concentrations do not change during aging like the stable precipitates or metallic compounds. Generally, from the viewpoint of stability, G. P. zones are classified as the metastable precipitates. However, Merz and Gerold ${ }^{(3)}$ revealed that the structure of the zones in the aluminum-rich aluminum-zinc alloys which were formed at room temperature did not change during aging. Besides, the constancy of the integrated intensity

(26) D. de Fontaine : Ph. D. Thesis, Northwestern University, (1967).
$Q_{0}$ as shown in Fig. 3 or in the previous results ${ }^{(20)(27)}$ confirms that the solute concentrations of G.P. zones and the matrix do not change after the aging time of $6 \mathrm{~min}$. Since the G.P. zones may be considered to be the "stable" precipitates from the viewpoint of stability, the Ostwald ripening can be applicable to the growth of G.P. zones and a plot of $\bar{R}^{3}$ vs. $t\left(>t_{0}\right)$ is given in Fig. 7 , where data are found to show the excellent linearity. The diffusion coefficient during coarsening of G.P. zones is.

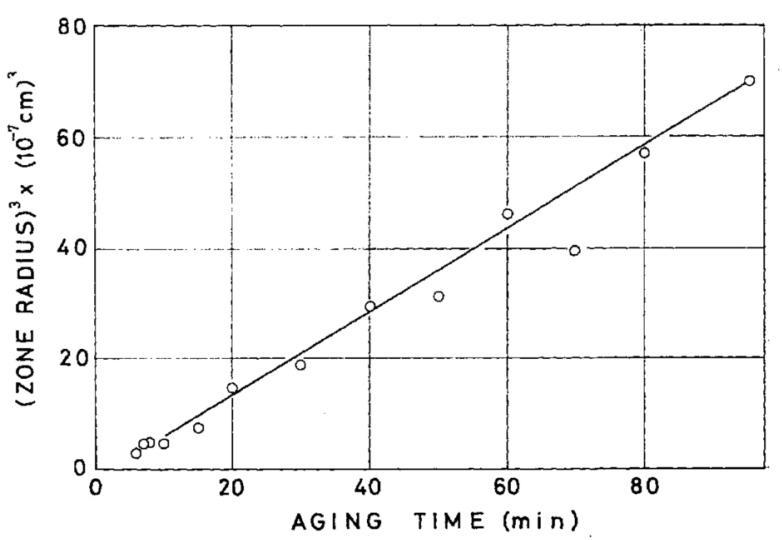

Fig. 7 Time dependence of the average zone radius for the alloys quenched from $300^{\circ} \mathrm{C}$ and aged at $40^{\circ} \mathrm{C}$.

estimated from the slope of the plot according to eq. (5 a). The measured value of $k$ for specimens aged for $6 \sim 95$ $\min$ is $5.069 \times 10^{-22} \mathrm{~cm}^{3} / \mathrm{sec}$. Putting $V_{m}=9.401 \mathrm{~cm}^{3} /$ g-atom, $T=313^{\circ} \mathrm{K}$, and $C_{e}=0.00185 \mathrm{~mol} / \mathrm{cm}^{3}$ into eq. (5 b), one obtains

$$
D \sigma=9.47 \times 10^{-11} \mathrm{erg} / \mathrm{sec} .
$$

To estimate the value of $D$ from this equation, since the value of $\sigma$ was not experimentally obtained at such low temperature, the calculated value, $\sigma=64 \mathrm{erg} / \mathrm{cm}^{2}$, was used by putting $H_{m}=232 \mathrm{cal} / \mathrm{mol}^{(28)}, N s=1.5 \times 10^{14} /$ $\mathrm{cm}^{2}$ and $Z s \simeq 4$ into eqs. (7) and (9). Then, from eq. (10) $D$ is calculated to be $1.5 \times 10^{-12} \mathrm{~cm}^{2} / \mathrm{sec}$. Using. the values of $D$ obtained above and $D_{0}=0.31 \pm 0.14$ $\mathrm{cm}^{2} / \mathrm{sec}^{(29)}$ which was determined by the use of the radioactive isotope $\mathrm{Zn}^{65}$, the activation energy $Q_{\mathrm{zn}_{\mathrm{n}}}$ for the diffusion of zinc atoms in aluminum is calculated to be $0.70 \pm 0.02 \mathrm{eV}$. From the temperature dependence of the critical time to reach electrical resistivity maximum, Panseri and Federighi ${ }^{(30)}$ obtained $Q_{\mathrm{zn}}=1.13 \pm 0.04 \mathrm{eV}$ in an $\mathrm{Al}-4.5$ at \% $\mathrm{Zn}$ alloy. Compared with this value, the one obtained by the present experiment is very low even though there is the difference in alloy concentrations. The origin of this discrepancy is not known, but. one of the significant reasons may be due to the difference of solute concentrations in the zones when the measurements were carried out. At the critical times

(27) M. Murakami, O. Kawano and Y. Murakami : Trans. Met. Soc. AIME, 245 (1969), 815.

(28) R. Hultgren, R. L. Orr, P.D. Anderson and K. K. Kelley : Selected Values of Thermodynamic Properties of Metals Alloy, John Wiley, New York, (1963), p. 274.

(29) J.E. Hilliard, B. L. Averbach and M. Cohen : Acta Met., 7 (1959), 86.

(30) C. Panseri and T. Federighi : Acta Met., 8(1960), 217. 
when the electrical resistivity measurements were carried out to determine the value of $Q_{\mathrm{z}_{n}}$, the decomposition seems not to be completed.

\section{Conclusions}

The measurements of the scattered intensities of the specimens quenched from $300^{\circ} \mathrm{C}$ and aged at $40^{\circ} \mathrm{C}$ by means of the X-ray small angle scattering method gave the information on the structures and the growth of G. P. zones in an Al-6. 8 at \% $\mathrm{Zn}$ alloy.

At the early stages of aging the formerly proposed models for the structures of zones were pointed out not to be consistent with the present experimental results and then an outline of a new structure for zones was given. At the later stages, when the solid solution was completely decomposed Gerold and Schweizer's model was indicated to be suitable for the structure of $G$. P. zones.

The kinetics of growth of these zones was proved to follow the Ostwald ripening and also the diffusion coefficient at later stages was calculated to be $1.5 \times 10^{-12}$ $\mathrm{cm}^{2} / \mathrm{sec}$ by applying the Lifshitz-Wagner theory to the zone growth.

\section{Acknowledgment}

The authors wish to express their bearty thanks to Professor D. de Fontaine for useful discussion and comments, and also wish to thank Professor H. Herman for reading the manuscript. 